\title{
Investigation of optimum wavelength converter based on nonlinear polarisation rotation in a bulk SOA
}

\author{
B.F. Kennedy, S. Philippe, F. Surre, A.L. Bradley and P. Landais
}

\section{Introduction}

All-optical signal processing continues to receive a lot of attention as a means of alleviating bottlenecks, due to electronic components, in optical communication systems. One of the most promising devices that has been put forward to perform this signal processing is the semiconductor optical amplifier (SOA) [1]. Techniques such as cross-gain modulation (XGM), cross-phase modulation (XPM) and four-wave mixing (FWM) continue to be developed. The polarisation dependence in the SOA is an obvious limitation of these techniques, but this effect can be exploited advantageously using cross-polarisation modulation (XPolM). This effect is based on nonlinear polarisation rotation (NPR). If a signal is injected into an SOA with a known state of polarisation it is possible to induce a nonlinear rotation of the output state of polarisation [2-7]. There are several causes of NPR. First, if a balance of the complex refractive index along the eigen axes is not achieved, then there is a polarisation dependence of the refractive index, confinement factor and modal gain. Second, tensile strain in the material can lead to a polarisation dependence of the material gain [8]. Recent reports suggest that the inherent strain in the device is sufficient to induce an asymmetric gain [9]. XPolM has been investigated as a technique to perform signal processing operations such as demultiplexing and wavelength conversion $[2,10,11]$. Wavelength conversion is the application which will be discussed in this paper. The intensity of a data signal is used to modulate the polarisation of a lowpower $\mathrm{CW}$ probe signal. This technique has previously been performed in both co- and counter-propagation setups $[10,11]$. However, no direct comparison of the performance can be concluded as the measurements were undertaken on different devices and under different conditions. For the reasons outlined above, the NPR is sensitive to device design and material strain. In order to develop a deeper understanding of XPolM in SOAs, results are presented in this paper in which an experimental comparison between both techniques is made, at a data rate of $2.5 \mathrm{~Gb} / \mathrm{s}$. Such a comparison has been presented for XPM but not for XPolM, to the best of the authors knowledge $[12,13]$. Wavelength conversion based on NPR has been presented in the literature at data rates of 5,10 and $40 \mathrm{~Gb} / \mathrm{s}[11,14,15]$. The focus of this paper is not the speed of conversion. However, the aim of this paper is to understand in greater detail the NPR effect which makes such wavelength conversion possible and will enable the optimisation of devices and conversion schemes exploiting XPolM. It is felt that this is of critical importance in order that the design of such wavelength converters be optimised. The underlying nonlinear behaviour, determined by the inter-band carrier dynamics, will be the same regardless of the bit rate.

The experimental setups for both co- and counterpropagation are detailed before the experimental results are presented. It is well established that counter-propagation techniques lead to a limitation in the maximum bit rate of wavelength conversion. This is due to the time required for the light to travel from one facet of the device to the other. However, one advantage of counter-propagation is that data may be converted to the same wavelength. This adds flexibility to the conversion scheme and also allows for pulse reshaping with SOAs. Prior to the analysis of the wavelength conversion, the level of the input probe power is varied in order to select its optimum value. An explanation of the importance of this selection is given. The results of the wavelength conversion are then discussed. An important point for XPolM is that XGM is inherent to the device and occurs simultaneously with XPolM. Results are presented for both inverted and noninverted XPolM, as well as for XGM. The relationship between the two effects is analysed in this paper. The wavelength dependence of the conversion is determined, in each case, over a span of $35 \mathrm{~nm}$. This span is limited by the operating range of the erbium doped fibre amplifier (EDFA), not by the SOA bandwidth. 


\section{Experimental setup}

Wavelength conversion is performed using XPolM at a data rate of $2.5 \mathrm{~Gb} / \mathrm{s}$ and a PBRS of $2^{7}-1$. This data rate is limited by the speed of the pseudo-random data generator and the error detector. The co-propagation setup is shown in Fig. 1. Two optical signals are simultaneously injected into the SOA. The SOA under test is an Avanex A1901, a tensile strained bulk amplifier structure with a gain spectral bandwidth of $75 \mathrm{~nm}$, centred at $1535 \mathrm{~nm}$. It is biased at $200 \mathrm{~mA}$ and temperature controlled at $23^{\circ}$. Both optical signals are generated using external cavity tunable lasers. The wavelength of the pump signal is $1538 \mathrm{~nm}$ and the wavelength of the probe signal is varied between 1535 and $1570 \mathrm{~nm}$. The span was limited to $35 \mathrm{~nm}$ due to the spectral bandwidth of the EDFAs, which operate in the C-band. The pump signal is modulated at $2.5 \mathrm{~Gb} / \mathrm{s}$ and then amplified through EDFA 2 to an average power of approximately $7 \mathrm{dBm}$ at the input of the SOA, which saturates the gain of the device. A Band-Pass Filter (BPF 2) is used to remove noise introduced by the EDFA. The probe signal is injected with a power of $-3.6 \mathrm{dBm}$. The signals are launched into the device in co-propagation using a 50:50 coupler.

The states of polarisation, of both the pump and probe signals, are controlled using polarisation controllers (PCs), which are adjusted in order to optimise the wavelength conversion. At the output of the SOA the combination of PC 3 and the PBS act to control the transmission of the amplified signal, based on the polarisation of the signal. It is the combination of these two components that distinguishes this setup from that of XGM. The PBS used has an extinction ratio of $20 \mathrm{~dB}$ and an insertion loss of $0.4 \mathrm{~dB}$. The wavelength conversion due to XPolM is based on the fact that the polarisation rotation is a nonlinear process. Both inverted and non-inverted XPolM can be performed using this setup by adjusting PC3 to maximise or minimise the transmission of the probe signal, in the absence of the pump signal. In the case of inverted XPolM, for example, the probe transmission is maximised before injection of the pump. It is necessary to include BPF 1 after the PBS in order to select the probe signal wavelength. This BPF has a $3 \mathrm{~dB}$ bandwidth of $1 \mathrm{~nm}$ and a wavelength tuning range of $40 \mathrm{~nm}$. EDFA 1 is used to amplify the wavelength converted signal and an attenuator is used to vary the received power falling on the photodetector. The signal can then be observed on an oscilloscope and Bit Error Rate Tester (BERT).

The counter-propagation setup is shown in Fig. 2. The operating conditions of the device and the pump and

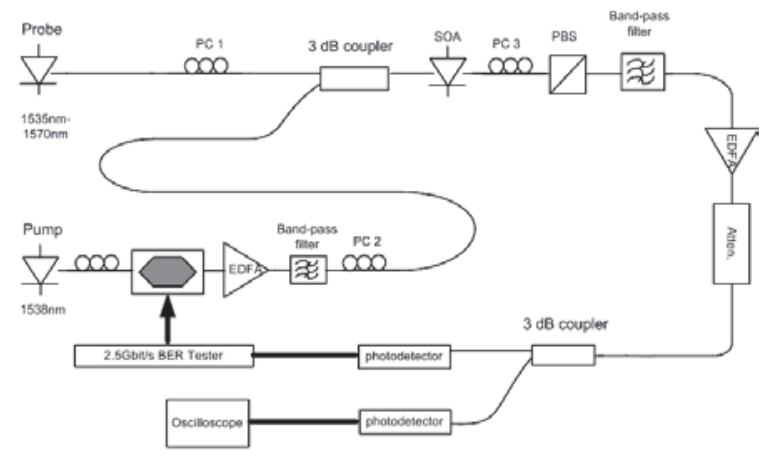

Fig. 1 Co-propagation XPolM experimental setup Bold lines represent electrical connections

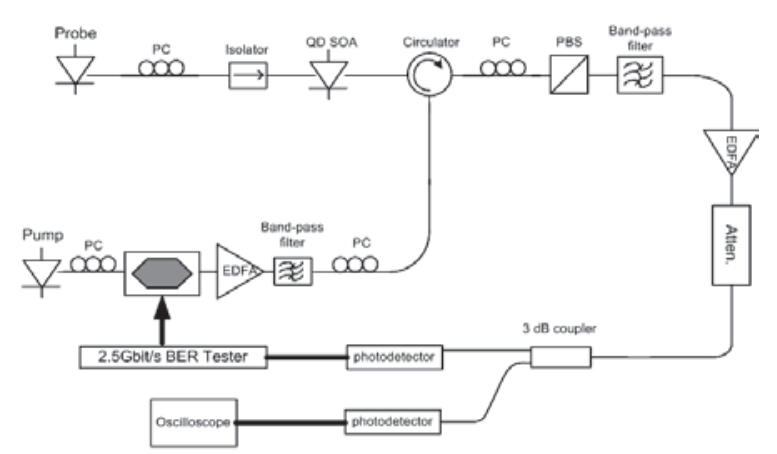

Fig. 2 Counter-propagation XPolM experimental setup Bold lines represent electrical connections

probe powers are the same as for the co-propagation setup. The pump and probe are injected into the device in opposite directions. A circulator with $35 \mathrm{~dB}$ isolation is used to inject the pump signal and to collect the converted signal. An isolator is used at the probe end of the device to ensure that the pump signal is not injected into the probe laser. Using this setup it is possible to convert data to the same wavelength. Because the signals are injected at opposite ends of the device it is not necessary to select the correct wavelength using BPF 1 but this filter is included to remove any ASE that may have been transmitted through the PBS. Other than the opposite propagation direction of the data signal, the co- and counter-propagation setups operate in the same manner. In order to make a comparison between both techniques it is important to ensure that the pump and probe signals are injected into the device with the same level of intensity. It should be noted that both co- and counter-propagation setups can be easily changed to a XGM setup by removing PC 3 and the PBS, respectively. The wavelength dependence of XPolM is determined in both experimental setups for inverted and non-inverted conversion. The probe signal is varied from $1535 \mathrm{~nm}$ to $1570 \mathrm{~nm}$, whereas the pump signal is maintained at $1538 \mathrm{~nm}$. The pump wavelength is fixed as its main function is to deplete the carrier density in order that the device operate in the nonlinear regime. The probe wavelength is varied in order to measure the range of operation of the wavelength conversion scheme.

\section{Results}

\subsection{Optimum level of input probe power}

The input probe power is a crucial parameter for XPolMbased wavelength conversion. Two extreme approaches have been taken in the literature, one in which the probe signal is minimised so that the gain of the device is not modulated by the probe [2-4] and the other in which the probe signal power is maximised [11]. To identify the optimum condition we studied the Q-factor as a function of injected probe power. The Q-factor is defined as:

$$
Q=\frac{I_{1}-I_{0}}{\sigma_{1}+\sigma_{0}}
$$

where $I_{1}$ and $I_{0}$ represent the mean signal level at ' 1 ' and ' 0 ', and $\sigma_{1}$ and $\sigma_{0}$ represent the standard deviation of the noise on the ' 1 ' and ' 0 ' level. It is measured using an oscillioscope. It should also be stated that the Q-factor is not directly related to the BER because of the non-Gaussian distribution of the intensity after the wavelength conversion. 


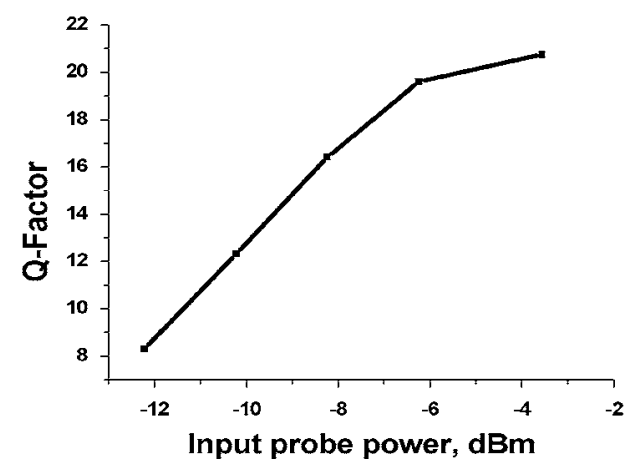

Fig. 3 Improvement of $Q$-factor as a function of injected probe power for non-inverted XPolM in counter-propagation

However, an approximation of the BER can be obtained from the Q-factor. The BER is measured in this experiment using a BERT as illustrated in Fig. 1 and Fig. 2. The results for co-propagation non-inverted XPolM are presented in Fig. 3. The $\mathrm{CW}$ probe power is varied from $-12.6 \mathrm{dBm}$ to $-3.6 \mathrm{dBm}$. The latter is the largest probe power that can be injected into the SOA, after all coupling losses have been considered, using the available tunable laser source. It may clearly be seen from Fig. 3 that there is a large increase in the Q-factor from 8 to 21 over this range. The result may be explained from observation of the polarisation resolved gain as a function of total injected $\mathrm{CW}$ power, shown in Fig. 4. As can be seen the polarisation discrepancy between TE and TM mode gain increases as a function of injected power. The fact that the biggest Q-factor is found when the probe signal power is largest is a consequence of the probe power coupling with the pump power to cause the greatest difference in gain along the eigen axes and causing a larger NPR. Therefore an injected power of $-3.6 \mathrm{dBm}$ is used for the rest of the paper. At this level of intensity there is a difference in gain along orthogonal axes of approximately $1.5 \mathrm{~dB}$, as can be observed in Fig. 4. The probe level of -3.6 was also found to be the optimum level, with the available equipment, for the counter-propagation setup. In this configuration the probe power was also varied, with the best results measured at $-3.6 \mathrm{dBm}$.

It may be seen that there is a discrepancy in Fig. 4. The TM gain is slightly larger than the TE gain for an injected power of approximately $-15 \mathrm{dBm}$. One would expect that the gain would be equal at this point. However, this polarisation dependence is within the polarisation sensitivity of

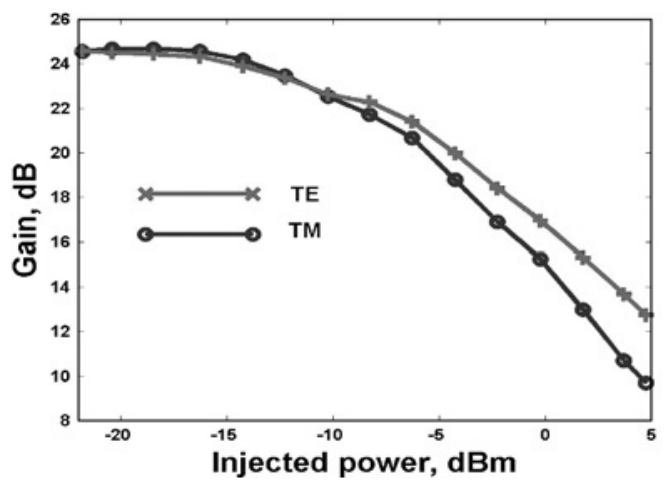

Fig. 4 Polarisation resolved gain as a function of injected power

the device, as provided by the manufacturers. The maximum polarisation sensitivity is given as $1.2 \mathrm{~dB}$.

The probe power of $-3.6 \mathrm{dBm}$ is the optimum level that can be obtained using the available equipment. However, if the probe power was increased beyond $-3.6 \mathrm{dBm}$ it is expected that a higher Q-factor could be obtained, as this would result in the SOA having a stronger NPR effect prior to injection of the pump. The true optimum value of the probe is that power which upon injection of the pump causes the device to reach the NPR saturation point that is the point where any increase in the injected power will not result in a larger discrepancy between the TE and TM gains. If the probe is increased beyond this optimum value then the level of the probe power is too large as it does not allow for the strongest NPR effect due to the pump power.

\subsection{Analysis of wavelength conversion}

The bit error rate (BER) as a function of received power for inverted and non-inverted XPolM, as well as XGM, is shown in Fig. 5 for both co- and counter-propagation schemes. The back-to-back data is also shown for comparison. In the co-propagation setup a penalty of approximately $1 \mathrm{~dB}$ is introduced for both inverted XPolM and XGM as can be seen in Fig. $5 a$. However, a larger penalty of approximately $5.5 \mathrm{~dB}$ is introduced using the non-inverted XPolM in the co-propagation setup. This poor performance is a consequence of XGM, which occurs simultaneously with XPolM. XGM leads to inverted conversion and therefore opposes the effect of non-inverted conversion because the converted signal experiences reduced gain. The opposite
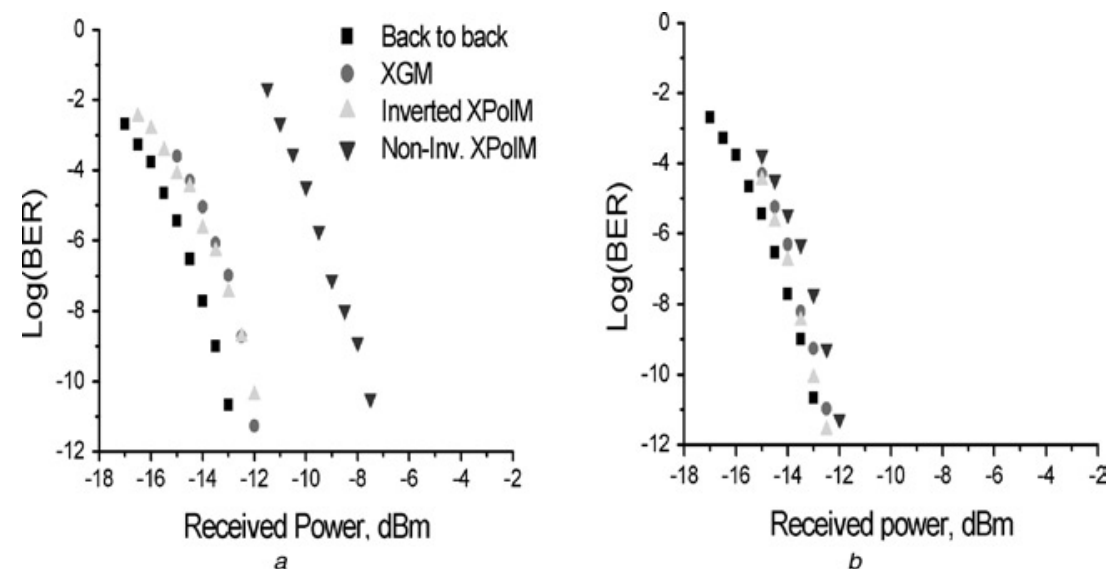

Fig. 5 BER as a function of received converted power for XGM, inverted XPolM and noninverted XPolM in a co- and $b$ counter-propagation.

Pump wavelength $=1538 \mathrm{~nm}$, probe wavelength $=1535 \mathrm{~nm}$ 

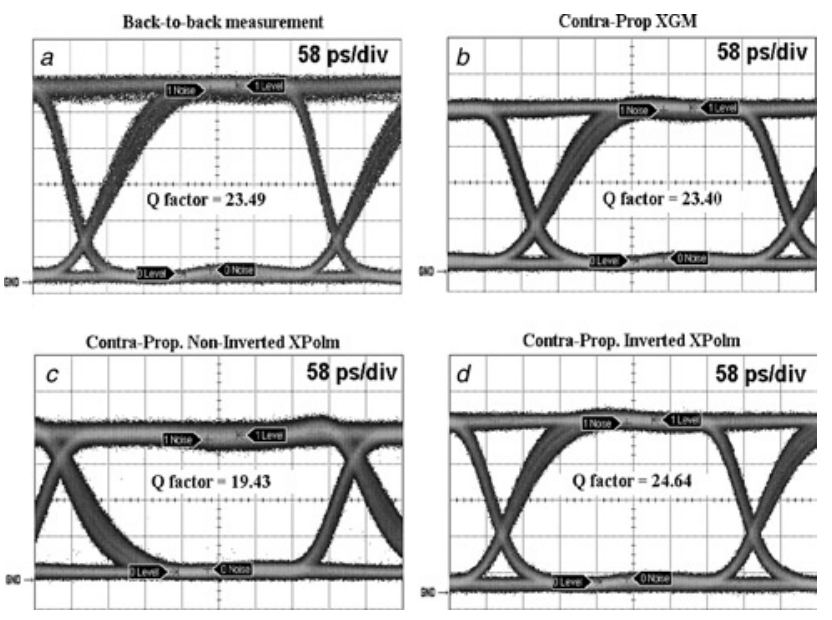

Fig. 6 Eye diagrams for back-to-back, XGM, non-inverted $X P o l M$ and inverted XPolM in counter-propagation

occurs for inverted XPolM, in which the signal inversion is enhanced by XGM. Fig. $5 b$ shows the results obtained from the counterpropagation setup. The penalty introduced to the inverted XPolM and XGM is reduced to approximately $0.5 \mathrm{~dB}$. This penalty is negligible if the insertion loss of the PBS in the counter-propagation setup is taken into consideration. However, the most significant variation between the co- and counter-propagation setups is in terms of the non-inverted XPolM. There is a reduction of approximately $4.5 \mathrm{~dB}$ in the power penalty for this technique between the two experimental setups. This result indicates that the NPR effect is present with less power in the counter-propagation configuration. This improvement in penalty was not found for inverted XPolM. This may be due to the PBS, which has an extinction ratio limited to $20 \mathrm{~dB}$. If the conversion has reached this limit then any improvement in the conversion, due to an increase in the NPR, will be undetected. The results presented in Fig. 5 are in contrast to those presented in [11], where the smallest power penalty was found to be in the non-inverted setup. This discrepancy may be due to the different relationships between the gain and polarisation nonlinearities in the two devices. It should be noted that although the results presented in Fig. 5 are in contrast with [11] they are in agreement with the results presented in [7].

The eye diagrams measured for the counter-propagation setup are shown in Fig. 6, along with the back-to-back measurement. The Q-factor measured for each setup is also shown. It can be seen that the Q-factor obtained for both inverted XPolM and XGM is practically the same as for the back-to-back case. From Fig. 6 it can be seen that a slow rising edge and a fast falling edge is present in the back-to-back eye diagram. It is logical to assume that the eye diagrams for the inverted configurations of XGM and inverted XPolM should therefore have a fast rising edge and a slow falling edge. Likewise, it is logical to assume the non-inverted XpolM configuration should have a slow rising edge and a fast falling edge, like the back-to-back eye diagram. However, from observation of Fig. 6 it appears that the opposite is true. This is caused by the presence of an inverted electrical amplifier used in the receiver. The pump power was also varied in both setups in order to optimise its value. The highest available pump power of $7 \mathrm{dBm}$ consistently led to the best XPolM performance. This is expected, once the device has not reached its NPR saturation point.

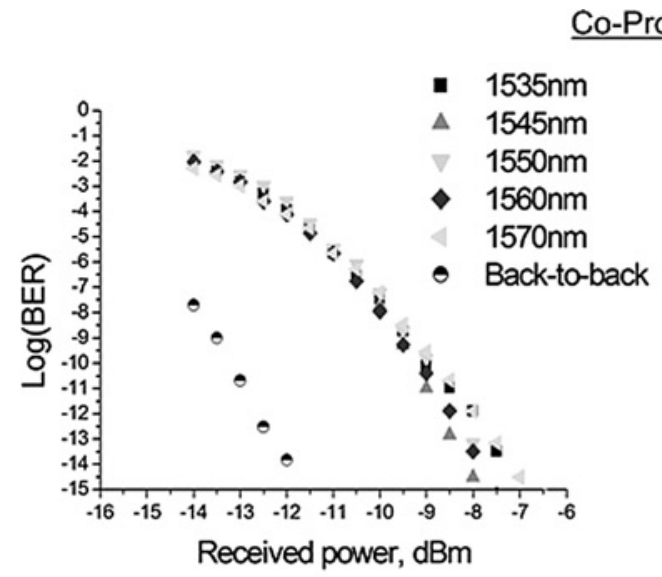

Co-Propagation

a

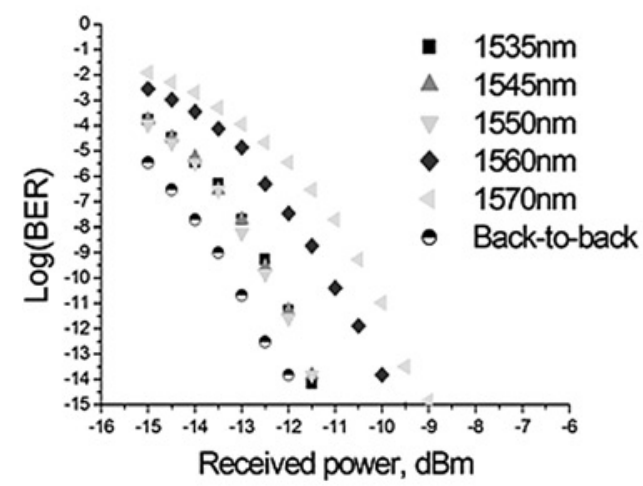

$d$

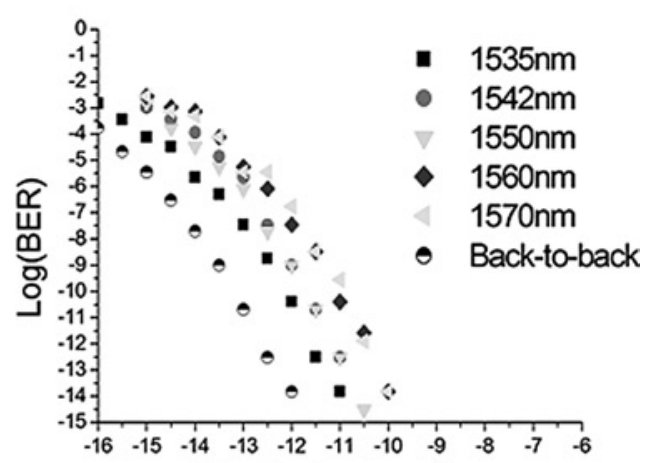

Received power, $\mathrm{dBm}$

Counter-Propagation

$b$

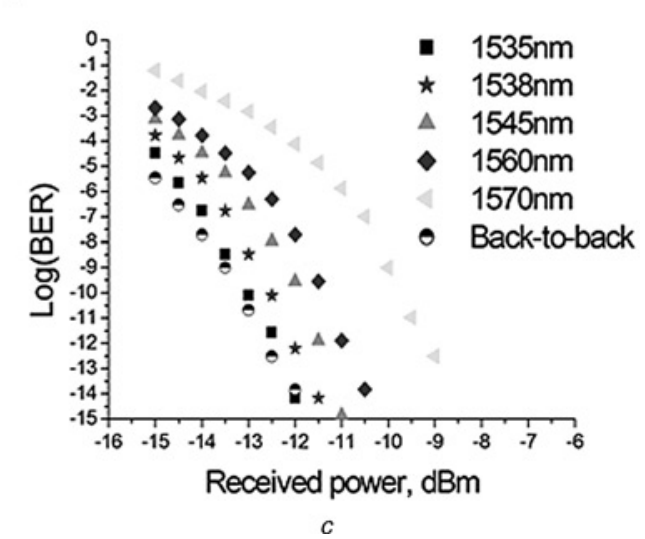

Fig. 7 BER as a function of received power for various probe signals wavelengths, where a-b represent non-inverted XPolM and $c-d$ represent inverted XPolM in co- and counter-propagation, respectively 


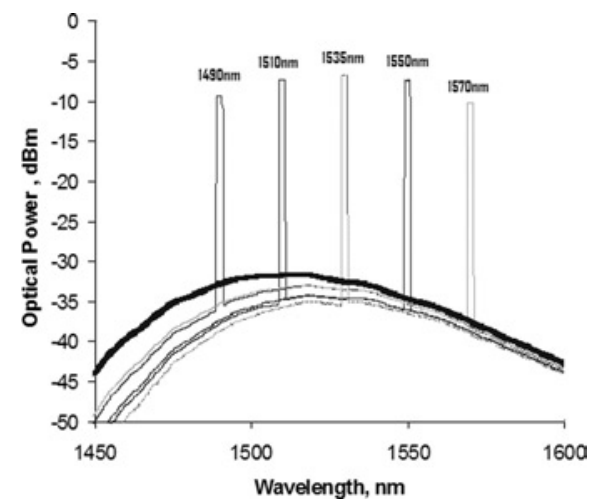

Fig. 8 Wavelength dependence of the gain suppression

\subsection{Wavelength dependence of the wavelength conversion}

The BER as a function of received power for various probe signal wavelengths is shown in Fig. 7. From this figure it can be seen that the effect of varying the probe wavelength is strongly dependent on the system configuration. Wavelength independent conversion is found for noninverted XPolM in the co-propagation setup, for BERs as low as $10^{-12}$, as can be seen from Fig. $7 a$. For BERs lower than this a small wavelength dependence is introduced. The cause of the insensitivity to wavelength may be explained from the relationship between XGM and XPolM. The wavelength dependence of the gain suppression can be seen from Fig. 8. At $1535 \mathrm{~nm}$, for example, there is a $3 \mathrm{~dB}$ suppression for a probe wavelength of $1535 \mathrm{~nm}$, whereas there is suppression of only $1 \mathrm{~dB}$ for a probe of $1570 \mathrm{~nm}$ at the same wavelength. It can be seen that the largest gain suppression occurs at $1535 \mathrm{~nm}$. As NPR is a nonlinear effect its strongest effect will also occur at the point of highest gain suppression. In the noninverted co-propagation setup the NPR acts to increase the extinction ratio of the converted signal. However, the suppressed gain causes a reduction of the extinction ratio, as it results in a lower amplification of the ' 1 ' level. As the probe wavelength is moved away from the gain peak of the SOA there are two effects on the wavelength conversion. Firstly, the NPR is reduced due to its intensity dependence. Secondly, the gain suppression is reduced. The result is that there is a balance in the contribution from these effects in the case of non-inverted XPolM in the co-propagation setup. Non-inverted XPolM is also obtained for the counter-propagation setup and is shown in Fig. 7b. It has already been established from Fig. 5 that the NPR effect is larger for counter-propagation, which results in a wavelength dependence of the BER as the NPR and gain suppression mechanisms are no longer balanced. The increase in NPR in the counter-propagation setup results in a much larger contribution of NPR than of
XGM. This leads to a wavelength dependence of $2.5 \mathrm{~dB}$ over the $35 \mathrm{~nm}$ span. Further comparison between Figs $7 a$ and $b$ reveal that the wavelength insensitivity in co-propagation is at the expense of a larger power penalty.

The wavelength dependence of inverted XPolM is also analysed. Obviously, wavelength insensitivity is not possible for inverted XPolM, as the NPR and gain suppression both increase the extinction ratio of the converted signal. In the co-propagation setup, as the wavelength is increased the power penalty increases by $1 \mathrm{~dB}$, as can be seen in Fig. 7c. Similarly, in counter-propagation, the power penalty over the same wavelength range increases by approximately $3.5 \mathrm{~dB}$ as shown in Fig. $7 d$. For both inverted and non-inverted conversion it can be seen that the wavelength dependence of the XPolM is smallest in co-propagation. In both cases the cause of the larger wavelength dependence in counter-propagation is the increase in the contribution of the wavelength dependent NPR effect.

The wavelength dependent power penalty introduced by both co- and counter-propagation setups, for non-inverted and inverted conversion, is presented in Table 1. For noninverted conversion in co-propagation, it can be seen that a wavelength dependence of just $0.2 \mathrm{~dB}$ is introduced over a $35 \mathrm{~nm}$ span. This is in contrast to inverted conversion in the counterpropagation setup where a wavelength dependence of $3.51 \mathrm{~dB}$ is introduced for a probe wavelength of $1570 \mathrm{~nm}$. The insensitivity to wavelength is at the cost of a penalty of over $5 \mathrm{~dB}$ whereas inverted conversion in the counter-propagation setup has a minimum penalty of $0.26 \mathrm{~dB}$. The same trends are observed in the analysis of the Q-factor as a function of probe wavelength. The Q-factor in the co-propagation setup, for non-inverted XPolM, and for a received power of $10^{-9}$ was measured as 6.4 at $1535 \mathrm{~nm}$. The value at $1570 \mathrm{~nm}$ was measured to be 6.2, a difference of only 0.2 in the Q-factor over a range of $35 \mathrm{~nm}$. In the non-inverted counter-propagation setup for the same experimental conditions a wavelength dependence in the Q-factor of 3.3 was measured over the same wavelength range. In each setup the highest Q-factor was measured for a probe wavelength of $1535 \mathrm{~nm}$.

\section{Conclusion}

Wavelength conversion using XPolM has been investigated. A comparison is made between co- and counter-propagation setups, in order to establish advantages and disadvantages of each. Both inverted and non-inverted XPolM were performed. Furthermore, a comparison was made with the performance of XGM wavelength conversion in the same device. It is shown that inverted XPolM and XGM yield a similar performance. The results indicate that the XPolM effect is larger in counter-propagation with a $4.5 \mathrm{~dB}$ improvement in the penalty introduced for non-inverted conversion. The wavelength dependence of the conversion is determined over a span of $35 \mathrm{~nm}$. It is shown that

Table 1: Power penalty calculated for non-inverted and inverted conversion, in both co- and counter-propagation schemes, for a BER of $10^{-9}$

\begin{tabular}{lllll}
\hline $\begin{array}{l}\text { Probe wavelength, } \mathrm{nm} \\
\text { Probe wavelength, } \mathrm{nm}\end{array}$ & $\begin{array}{l}\text { Co-propagation } \\
\text { Non-inverted, } \mathrm{dB}\end{array}$ & $\begin{array}{l}\text { Co-propagation } \\
\text { Inverted, } \mathrm{dB}\end{array}$ & $\begin{array}{l}\text { Counter-propagation } \\
\text { Non-inverted, } \mathrm{dB}\end{array}$ & $\begin{array}{l}\text { Counter-propagation } \\
\text { Inverted, } \mathrm{dB}\end{array}$ \\
\hline 1535 & 5.21 & 1.26 & 1 & 0.26 \\
1550 & 5.11 & 1.51 & 1.06 & 1.41 \\
1560 & 5.11 & 2.26 & 2.26 & 2.51 \\
1570 & 5.31 & 2.26 & 2.91 & 3.51 \\
\hline
\end{tabular}


wavelength insensitive conversion is possible for noninverted XPolM in the co-propagation setup over the accessible wavelength range, although a larger penalty is introduced in this case. The cause of this wavelength insensitivity is attributed to a balancing between NPR and gain suppression in the non-inverted case. It may be concluded that the question of whether to use a co- or counterpropagation setup for XPolM is dependent on the exact requirements of the system. If wavelength insensitivity is desired then co-propagation is more suitable, whereas if the power penalty is a crucial parameter then counterpropagation should be used.

\section{References}

1 Cotter, D., Manning, R.J., Blow, K.J., Ellis, A.D., Kelly, A.E., Nesset, D., Phillips, I.D., Poustie, A.J., and Rogers, D.C.: 'Nonlinear optics for high-speed digital information processing', Science, 1999, 286, pp. $1523-1528$

2 Patrick, D.M., Ellis, A.D., Davies, D.A.O., Tatham, M.C., and Sherlock, G.: 'Demultiplexing using polarization rotation in a semiconductor laser amplifier', Electron. Lett., 1994, 30, pp. 341-342

3 Diez, S., Schmidt, C., Ludwig, R., Weber, H.G., Doussiere, P., and Ducellier, T.: 'Effect of birefringence in a bulk semiconductor optical amplifier on four-wave mixing', IEEE Photon. Tech. Lett., 1998, 10, pp. $212-214$

4 Soto, H., Erasme, D., and Guekos, G.: 'Cross polarization modulation in semiconductor optical amplifiers', IEEE Photon. Tech. Lett., 1999, 11, pp. 970-972

5 Manning, R.J., Antonopoulos, A., Le Roux, R., and Kelly, A.E.: 'Experimental measurement of nonlinear polarization rotation in semiconductor optical amplifiers', IEE Electron. Lett., 2001, 37, pp. $229-231$
6 Kennedy, B.F., Philippe, S., Lais, P., Bradley, A.L., and Soto, H. 'Experimental investigation of polarization rotation in SOAs', IEE Proc.-Optoelectron., 2004, 151, pp. 114-118

7 Guo, L., and Connelly, M.J.: 'Signal induced birefringence and dichroism in a tensilestrained bulk semiconductor optical amplifier and its application to wavelength conversion', IEEE J. Light. Technol., 2005, 23, pp. 4037-4045

8 O'Reilly, E.P., and Adams, A.R.: 'Band-structure engineering in strained semiconductor lasers', IEEE J. Quant. Elect., 1994, 30, pp. $366-379$

9 Wang, W., Allaart, K., and Lenstra, D.: 'Semiconductor optical amplifier gain and anisotrophy', Electron. Lett., 2004, 40, pp. $1602-1603$

10 Stephens, M.F.C., Asghari, M., Penty, R.V., and White, I.H.: 'Demonstration of ultrafast all-optical wavelength conversion utilizing birefringence in semiconductor optical amplifiers', IEEE Photon. Tech. Lett., 1997, 9, pp. 449-451

11 Liu, Y., Hill, M.T., Tangdiongga, E., deWaardt, H., Calabretta, N., Khoe, G.D., and Dorren, H.J.S.: 'Wavelength conversion using nonlinear polarization rotation in a single semiconductor optical amplifier', IEEE Photon. Tech. Lett., 2003, 15, pp. 90-92

12 Bischoff, S., Buxens, A., Fischer, S.T., Dulk, M., Clausen, A.T., Poulsen, H.N., and Mørk, J.: 'Comparison of all-optical co- and counter-propagating high-speed signal processing in SOA-based Mach-Zehnder interferometers', Kluwer Opt. Quant. Elec., 2001, 33, pp. $907-926$

13 Nishimura, K., Usami, M., Asai, T., and Utaka, K.: 'Dependence of efficiency ratio of XPM to XGM on control pulse propagation direction in SOA'. Optical Amplifier and their Applications, Paper WD4, Budapest, August 2005

14 Soto, H., Erasme, D., and Guekos, G.: '5-Gb/s XOR optical gate based on crosspolarization modulation in semiconductor optical amplifiers', IEEE Photon. Tech. Lett., 2001, 13, pp. 335-337

15 Contestabile, G., Calabreta, N., Presi, M., and Ciaramella, E.: 'Single and multicast wavelength conversion at $40 \mathrm{~Gb} / \mathrm{s}$ by means of fast nonlinear polarization switching in an SOA', IEEE Photon. Tech. Lett., 2005, 17, pp. 2652-2654 